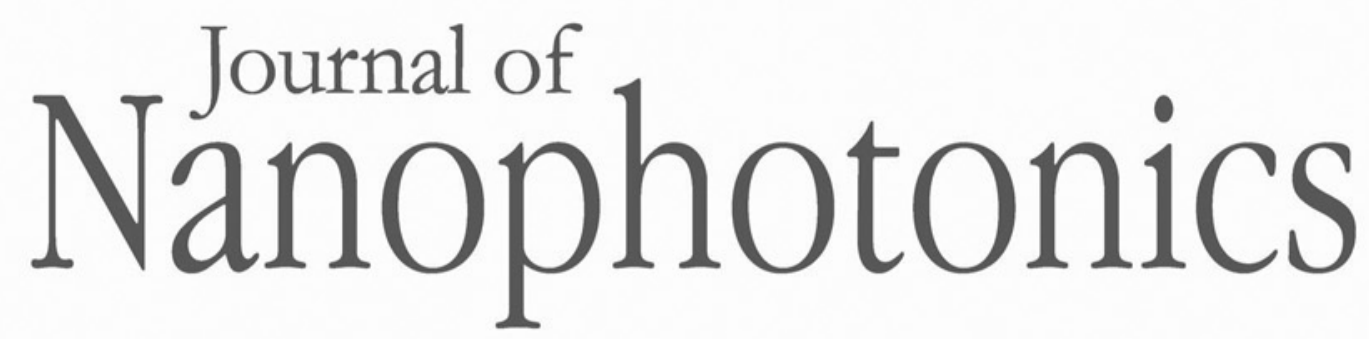

\title{
Laser beam scanning using near-field scanning optical microscopy nanoscale silicon-based photodetector
}

Matityahu Karelits

Yaakov Mandelbaum

Avraham Chelly

Avi Karsenty 


\title{
Laser beam scanning using near-field scanning optical microscopy nanoscale silicon-based photodetector
}

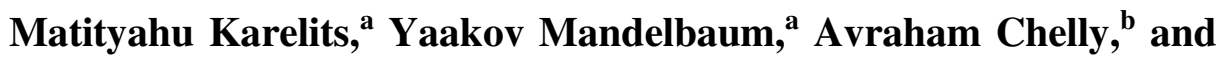 \\ Avi Karsenty ${ }^{\mathrm{a}, *}$ \\ ${ }^{a}$ Lev Academic Center, Advanced Laboratory of Electro-Optics (ALEO) Simulations and \\ Characterization, Department of Applied Physics/Electro-Optics Engineering, Jerusalem, Israel \\ ${ }^{b}$ Bar-Ilan University, Faculty of Engineering, Ramat Gan, Israel
}

\begin{abstract}
As part of the efforts to enhance the near-field scanning optical microscopy and the detection of evanescent waves, a silicon Schottky diode, shaped as a truncated trapezoid photodetector and sharing a subwavelength pin-hole aperture, has been designed and simulated. Using finite elements method and two-dimensional advanced simulations, the detector has been horizontally shifted across a vertically oriented Gaussian beam, which is projected on top of the device. Both electrical and electro-optical simulations have been conducted. These results are promising toward the fabrication of a new generation of photodetector devices. (C) The Authors. Published by SPIE under a Creative Commons Attribution 3.0 Unported License. Distribution or reproduction of this work in whole or in part requires full attribution of the original publication, including its DOI. [DOI: 10.1117/1.JNP.12.036002]
\end{abstract}

Keywords: near-field scanning optical microscopy; silicon; photodetector; pin-hole subwavelength aperture; evanescent waves; finite elements method; two-dimensional simulations.

Paper 18023 received Feb. 14, 2018; accepted for publication Jul. 11, 2018; published online Jul. 23, 2018.

\section{Introduction}

The near-field and subwavelength aperture interactions have been largely studied during the last years, ${ }^{1}$ as well as the use of relevant photodiodes. ${ }^{2-4}$ In such conditions, computer-aided design modeling becomes more necessary. The finite elements method (FEM) has been proven useful in the development of advanced nanoscale devices. ${ }^{5,6}$ The general framework of FEM discretization, meshing and solver algorithms together with techniques for dealing with challenges such as multiple time scales, shocks, and nonconvergence, is well known; these include load-ramping, segregated iterations, and adaptive meshing. Recently, this method, based on mathematical models and using optimized mesh structures, has enabled the performance of advanced simulations of a photodiode with subwavelength aperture. ${ }^{7-9}$ Installation of such nanoscale electro-optical sensors on top of existing microprobes and cantilevers may bring higher performance to nearfield optics research by creating a new generation of advanced near-field scanning optical microscopy (NSOM). In the last decades, both atomic force microscope (AFM) and scanning tunneling microscope have made significant progress in the fabrication of their own tips. Promising results optical imaging was reported, using a side pin-hole photodetector combined with a tip ${ }^{10}$ or UV NSOM. ${ }^{11}$

In Ref. 9, an initial three-dimensional (3-D) analysis of a conical NSOM detector was presented. Basic electro-optic properties such as the $I-V$ curve and optical responsivity were presented. The construction and potential application of the device were discussed, together with a detailed description of the simulation. A theoretical discussion of evanescent waves and subwavelength resolution appears there too.

However, where semiconductor modeling is involved, the nonlinear nature of the driftdiffusion equations means that full 3-D modeling of imaging is still a computational challenge.

*Address all correspondence to: Avi Karsenty, E-mail: karsenty@jct.ac.il 
Instead a two-dimensional (2-D) model can be used to simulate a device whose orthogonal dimension is significantly larger than its lateral dimensions-rods, wires, etc. In this paper, we present a 2-D simulation of a trapezoid-shaped NSOM detector with a slit aperture whose out-of-plane dimension is much larger than the cross-sectional dimension. The COMSOL Multiphysics software package, based on the FEM, was employed. The device consists in a silicon Schottky photodiode bearing a subwavelength top aperture. Such a nanoscale electrooptical sensor, placed on an AFM cantilever's edge, would insure collection of the topography and the optical data. The electric response, while scanning a laser beam, was studied and optimized by changing both device related specification. It is shown that high resolution of the order of the wavelength is obtained. This is useful as a validation of the behavior of the device in comparison to current standards and benchmarks.

\section{Simulation Method}

A detailed description of the simulation was presented in Ref. 9 for the 3-D pyramidal device. The 2-D simulation is similarly performed; hence, the technical description is brought in this section. Here, we discuss the few selected points, which are specific to the 2-D simulation.

\subsection{Two-Dimensional Simulation Conditions and Process}

Though 3-D simulations are ideal, many 2-D simulations have been performed in the past due to constraints of computing power. ${ }^{12-16}$ Sometimes, the applicability of the results was understood to be limited to the qualitative aspects. However, in this work, we analyze the behavior of a device whose orthogonal dimension is significantly larger than its lateral dimensions, so that a 2-D model is more than sufficient to simulate its behavior. This detector has a large extent in the $z$-direction, orthogonal to the plane of the simulation. Its aperture is thus a slit-subwavelength in the plane of the simulation, unconfined in the orthogonal direction. Accordingly, the impinging radiation is incident in the plane of the radiation; the Gaussian beams employed are 2-D Gaussian beam.

The simulation was performed with the COMSOL Multiphysics, which uses the FEM. The simulation couples the semiconductor and the wave optics modules using the optoelectronics interface.

The semiconductor module computes the electronic transport and charge distribution within the silicon using the drift-diffusion equations for the electron and hole densities in the semiconductor coupled to Poisson's equation for the electric "band-bending" potential. These are augmented by statistical mechanics to describe the effect of doping on the carrier charge population. Light-matter interactions, described ahead, occur primarily within the Schottky junction, which develops between the semiconductor bulk and metal surface. The junction is a depletion layer, which develops in the semiconductor due to the difference in Fermi levels between the two materials. The work function of the metal, the electron affinity of the semiconductor, and the doping level are input parameters. In addition, the phenomenon of thermionic emission from the metal to the semiconductor is built as a boundary condition on the driftdiffusion current, which determines the boundary conditions on the charge distribution.

The material chosen for the interior of the device was silicon, n-doped to a donor concentration $N_{d}=10^{17} \mathrm{~cm}^{-3}$ using analytic doping. Trap-assisted recombination was activated, with default values. The sides of the device serve as the Schottky junction. This is realized using the metal contact boundary condition with the rectifying junction option selected, together with "thermionic emission." The material chosen for the model was aluminum, which sets the work function, and the external bias was set to the appropriate voltage. Together these determine the boundary condition for the electric potential $\phi$

$$
q V=E_{F, m}=q \phi-\Phi_{m},
$$

where $\Phi_{m}$ is the metal work function and $V$ is the external bias. The Schottky junction only extends along the upper section of the device. The rest of the exterior is given an insulating boundary condition. This defines the boundary conditions relative to the semiconductor module. 
The wave optics module simulates the propagation of the electromagnetic wave using Maxwell's equations. On the entire outer surface of the device (excluding the ends), a perfect electric conduction boundary condition was used, which imposes a Dirichlet condition on the component of the electric field parallel to the surface. This affects a perfectly reflecting boundary, i.e., a perfect metal. This is a good approximation, as the entering electromagnetic wave decays entirely in the aluminum before penetrating through to the silicon in the interior. In order to simulate photodetection, "optical transitions" were activated in the interior. This adds a photogeneration source term to the convection-diffusion equations, which is proportional to the intensity of the electromagnetic field. The interaction between the radiation and the siliconphotodetection-involves indirect and direct interband transitions. The precise form of this term is determined by Fermi's golden rule, together with an empirical model for indirect absorption in silicon, based on an empirical model of Green and Keevers. ${ }^{17}$

The incoming optical excitation is affected at the upper surface using a port boundary condition with the relevant value of the electromagnetic intensity. The lower surface is given an open boundary condition using the scattering boundary condition selection. Finally, with regards to the electric potential, the upper surface is given an insulating boundary condition, whereas the lower surface is defined as a metal contact boundary condition with the ohmic contact option.

\subsection{Polarization Aspects}

This work reports on an incident transverse electric (TE) wave linearly polarized in the out-ofplane direction. This restriction from the longitudinal polarization is a consequence of the large extent of the device in this direction-absorption of this polarization is significantly stronger than of waves polarized in the direction transverse to the slit. This is a familiar result from the interaction of EM waves with wires and nanorods, ${ }^{18}$ where it serves as the basis for design of many polarizers.

\subsection{Metallic Boundaries and Surface Polariton-Plasmon Resonances}

The exterior walls of the device are composed of a layer of aluminum (Fig. 1). In the upper section of the device, this layer serves as the anode of the Schottky junction. In the simulation, a perfect metal was assumed, i.e., a perfect electric conduction boundary condition was used, which imposes a Dirichlet condition on the component of the electric field parallel to the surface. Regarding the semiconductor component, the metal-semiconductor junction is modeled as a boundary condition. The metal component itself has no thickness and does not model internal bulk currents.
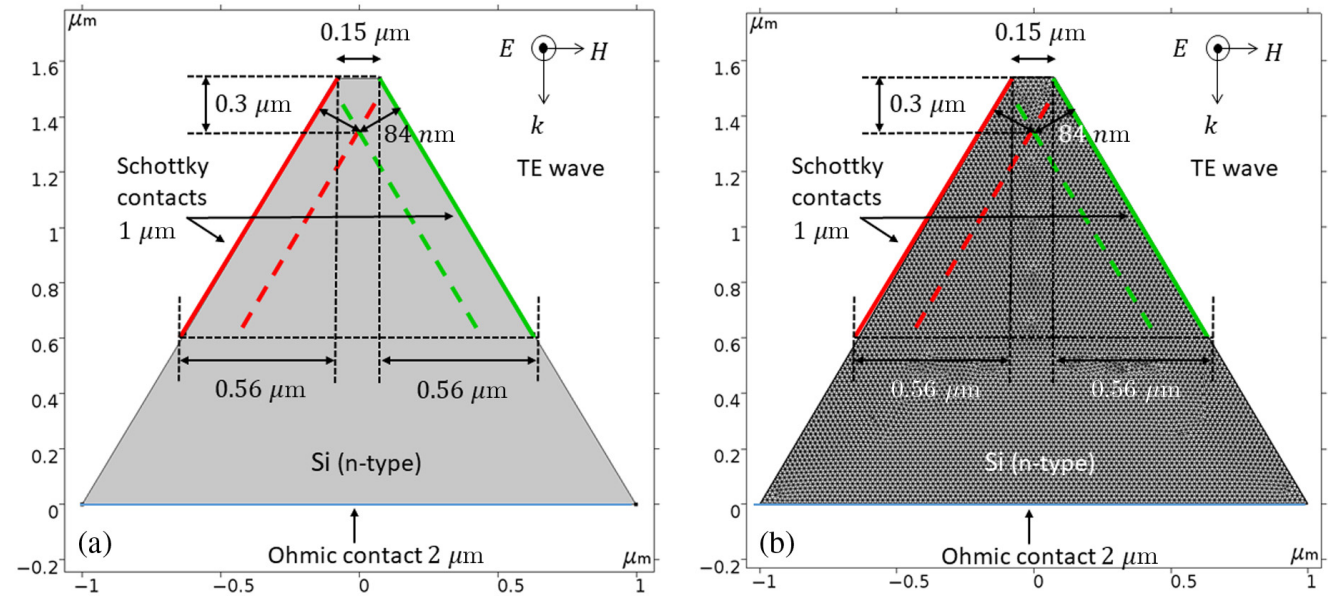

Fig. 1 COMSOL simulation of the NSOM photodetector structure. (a) The depletion layer boundaries are marked by red dashed lines for a given bias of $-0.5 \mathrm{~V}$ applied at the Schottky contact. The TE wave components are indicated in the inset. (b) COMSOL simulation with the mesh structure. 
Regarding the possibility of surface polariton-plasmon (SPP) resonances at the insulator to metal interface, we can argue that only transverse magnetic (TM) electromagnetic waves can excite such resonances. Assuming that the metal boundaries are modeled as perfect conductors, this translates into the Dirichlet boundary condition on the electric field: $E_{\|}=0$. This in turn corresponds to zero impedance and vanishing skin depth, so that the electric field is entirely excluded from the interior of the metal. In this limit, the conductance is infinite so that the complex dielectric function is also infinite according the Drude equation ${ }^{19}$

$$
\epsilon=\epsilon_{\text {real }}+j \frac{\sigma}{\omega} \text {. }
$$

The SPP wave number ${ }^{18}$ is then reaching the total wavenumber $k$ according

$$
k_{\|}=\frac{\omega}{c} \sqrt{\frac{\epsilon \epsilon_{r}}{\epsilon+\epsilon_{0} \epsilon_{r}}} \stackrel{\epsilon \vec{\infty}}{\rightarrow} \frac{\omega}{c} \sqrt{\epsilon_{r}}=n \frac{\omega}{c}=k,
$$

where $\epsilon_{r}$ is the dielectric constant of the surrounding dielectric and $n$ is the index of refraction. This implies the nullity of the normal component $k_{\perp}$. Combined with the requirement above that the electric field is orthogonal to the surface, this leads to the conclusion that only beams at perfectly glancing incidence can excite an SPP on the metal surface. The Gaussian beam propagates along the negative $y$-axis but includes plane wave components along the other direction. These are distributed in $k$-space (the "angular distribution") with a Gaussian distribution of width inverse to the waist. Due to the sloping sides of the device, glancing incidence, like other off-axis component directions, is suppressed. On the other hand, the absorbance peak of a surface plasmon is quite narrow, ${ }^{18}$ with regard to the angle of incidence, even for a real metal with nonzero dissipation. Thus the radiation absorbed in the SPP channel, obtained by integrating the product of the EM wave distribution with the SPP resonant peak, is expected to be quite small. In summary, the TE polarization is the dominant channel for electromagnetic interactions for this device architecture.

\section{Electro-Optical Simulations Results}

\subsection{Two-Dimensional Scanning of Laser Beam Illumination}

The NSOM detector is simulated in 2-D as a trapezoidal structure of $1.54-\mu \mathrm{m}$ height, $2-\mu \mathrm{m}$ bottom width, and 150-nm top aperture diameter. As shown in Figs. 2 and 3, the device-identifiably by its trapezoid silhouette-is shifted horizontally across the vertical projected Gaussian laser beam of 550-nm wavelength having a $0.69-\mu \mathrm{m}$ full width at half maximum (FWHM). The color scale indicates the beam intensity as the electric field value (V/m). In Fig. 2, the device is presented at its starting point, on the left side of the beam. The scanning proceeds when the device is shifting to the right direction. In Fig. 3, the device's position approaches the center of the beam where it absorbs part of the intensity.

Our analysis consists in varying several parameters in order to optimize the photocurrent response. The metal contact is $1-\mu \mathrm{m}$ height from the top aperture (both sides of the trapezoid). The ground electrode is at the bottom of the structure. The first class of parameters we will consider is the device intrinsic specifications such as the silicon doping level and the metal work function. Then, we will consider the influence of external parameters such as the bias voltage, the radiant intensity, and the laser wavelength.

\subsection{Photocurrent Detection Resolution}

As discussed above, the FWHM of the impinging beam is $0.69 \mu \mathrm{m}$. In comparison, Fig. 4 shows that the FWHM of the signal detected by the device is $\sim 0.77 \mu \mathrm{m}$. This reflects geometric averaging due to the finite width of the aperture - the FWHM is increased by an amount 1 to 2 times the width, which is $150 \mathrm{~nm}$ in this case. It is to be stressed that the increased FWHM of the signal is geometric and is less than that would be expected due to diffraction. 


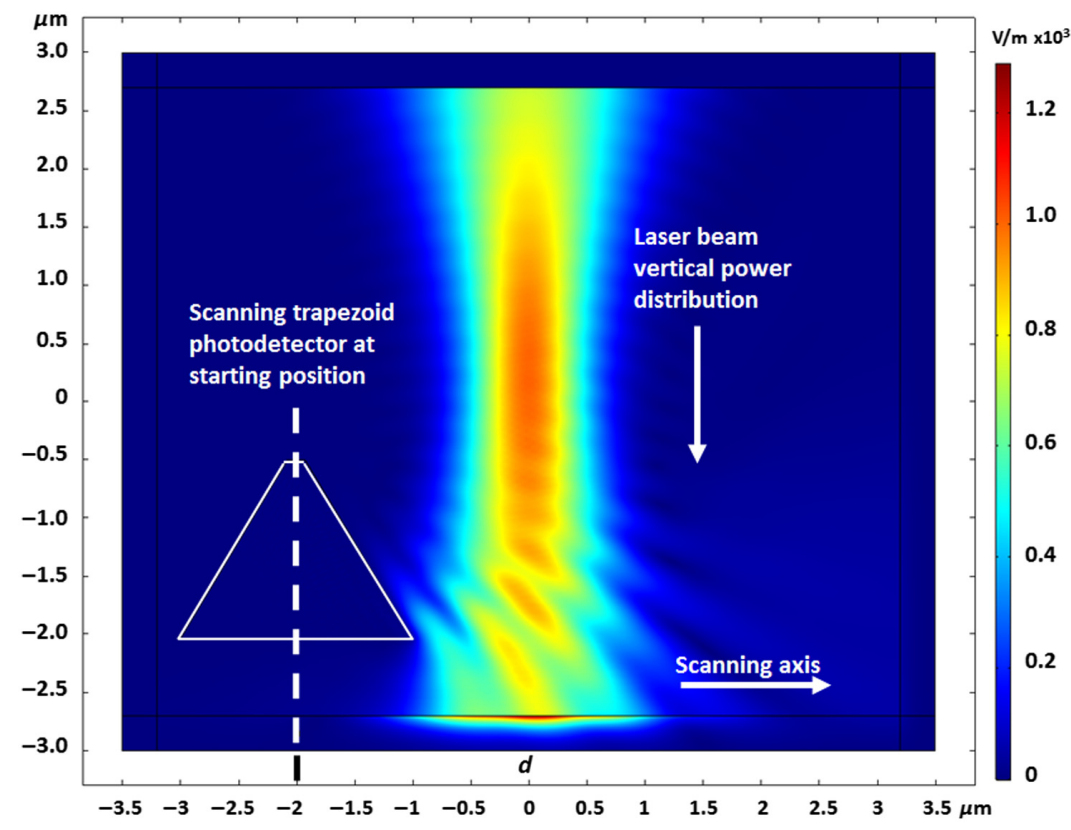

Fig. 2 Initial conditions of the scanning process, with the device located on the left side of the laser beam, incoming from above. The Gaussian laser beam has an FWHM of $1.1 \mu \mathrm{m}$. The distance from the top center of the photodetector to the beam center is $2 \mu \mathrm{m}$.

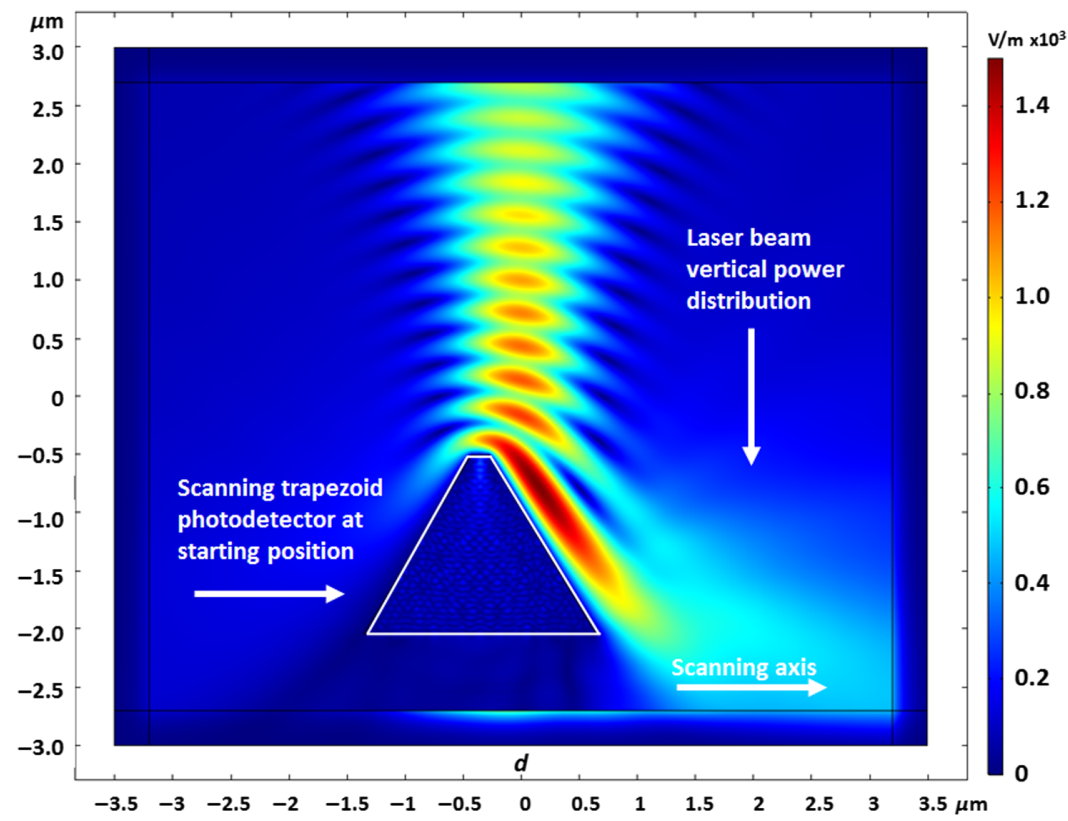

Fig. 3 Simulation result of the beam scanning, using a shifted trapezoid moving horizontally under the vertical illumination. The distance from the top center of the photodetector to the beam center is $0.5 \mu \mathrm{m}$.

\subsection{Photocurrent Scan as a Function of Silicon Doping}

Figure 4 shows the simulation of the current measured at the metal electrode by scanning the detector across the laser beam as described above. The varying parameter is the doping level of the n-type silicon. The simulation was performed under the following conditions: the metal work function is $4.72 \mathrm{eV}(\mathrm{Al})$, wavelength $\lambda$ is $550 \mathrm{~nm}$, the voltage bias is $-0.5 \mathrm{~V}$, and the electric field is $10^{3} \mathrm{~V} / \mathrm{m}$ equivalents to a radiant intensity is $0.15 \mathrm{~W} / \mathrm{m}^{2}$. The doping is reduced here from $10^{18}$ to $10^{16} \mathrm{~cm}^{-3}$. An enhancement of the simulated current is obtained. 
Karelits et al.: Laser beam scanning using near-field scanning optical microscopy...

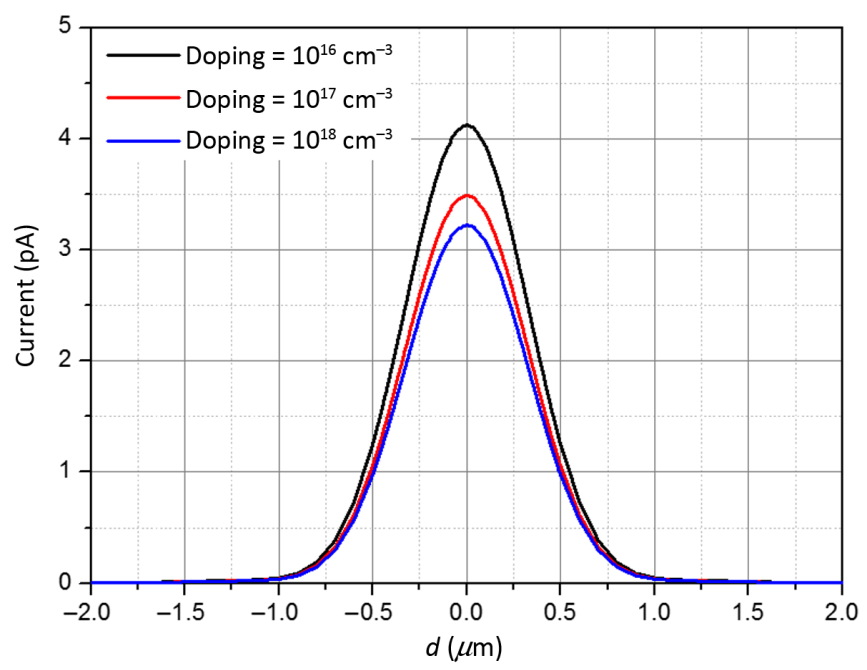

Fig. 4 Current as a function of the photodetector's position $(\mu \mathrm{m})$, relative to the beam center, for several doping values. The work function is $4.72 \mathrm{eV}(\mathrm{Al}), \lambda$ is $550 \mathrm{~nm}$, the voltage bias is $0 \mathrm{~V}$, and the radiant intensity is $0.15 \mathrm{~W} / \mathrm{m}^{2}$.

\subsection{Photocurrent Scan as a Function of Metal Work Function}

In order to enhance the applicability of the study, the simulations were performed for several contact metals. The considered elements are: aluminum (Al), tungsten (W), and gold (Au) with respective work function values of $4.72,4.97$, and $5.82 \mathrm{eV}$. The scanning of the laser beam in these conditions is presented in Fig. 5.

\subsection{Photocurrent Scan as a Function of Bias Voltage}

In Fig. 6, the bias voltage has been changed from 0 to $-5 \mathrm{~V}$ in steps of $-0.5 \mathrm{~V}$. The simulation was performed under the following conditions: silicon doping is $10^{17} \mathrm{~cm}^{-3}$, metal work function is $4.72 \mathrm{eV}(\mathrm{Al}), \lambda$ is $550 \mathrm{~nm}$, and the radiant intensity is $0.15 \mathrm{~W} / \mathrm{m}^{2}$.

We note here two contributions for the electric current, which are varying with the bias voltage: the minimum value attributed to the dark current $I_{\text {dark }}$ (taken for example at $d=-2 \mu \mathrm{m}$ ), and the maximum value $I_{\max }$ corresponding to the laser beam center $(d=0 \mu \mathrm{m})$. In order to

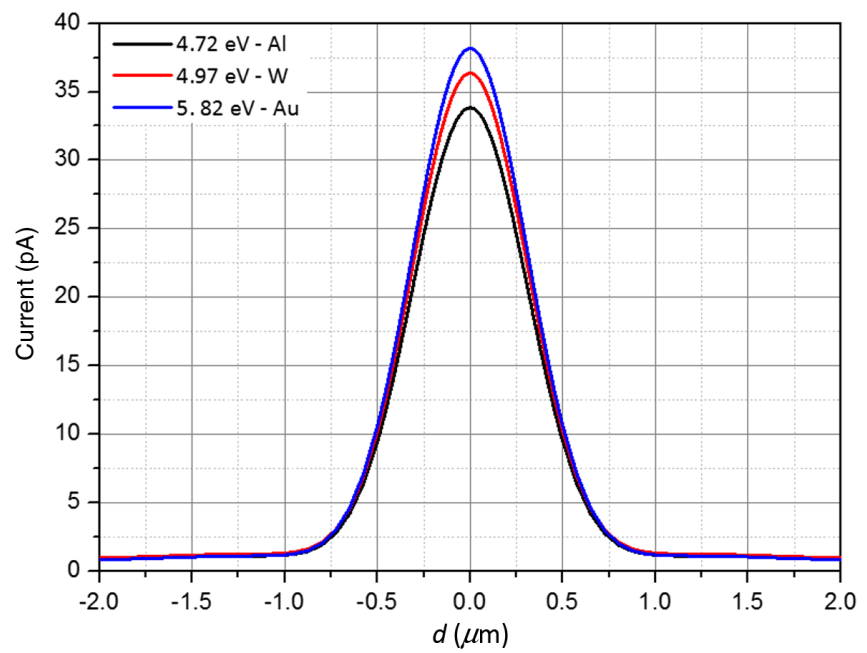

Fig. 5 Current as a function of the photodetector's position $(\mu \mathrm{m})$, relative to the beam center, for several work function values. The simulation was performed under the following conditions: doping is $10^{17} \mathrm{~cm}^{-3}, \lambda$ is $550 \mathrm{~nm}$, voltage bias is $-0.5 \mathrm{~V}$, and radiant intensity in this case is $1 \mathrm{~W} / \mathrm{m}^{2}$. 


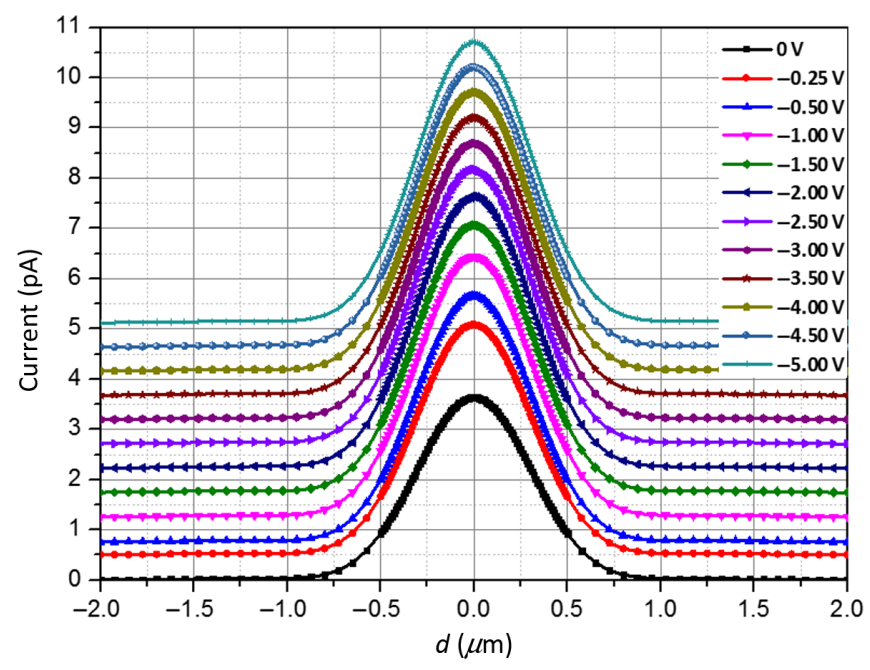

Fig. 6 Current as a function of the photodetector's position $(\mu \mathrm{m})$, relative to the beam center, for several bias voltage values. Doping is $10^{17} \mathrm{~cm}^{-3}$, work function is $4.72 \mathrm{eV}(\mathrm{Al}), \lambda$ is $550 \mathrm{~nm}$, and radiant intensity is $0.15 \mathrm{~W} / \mathrm{m}^{2}$.

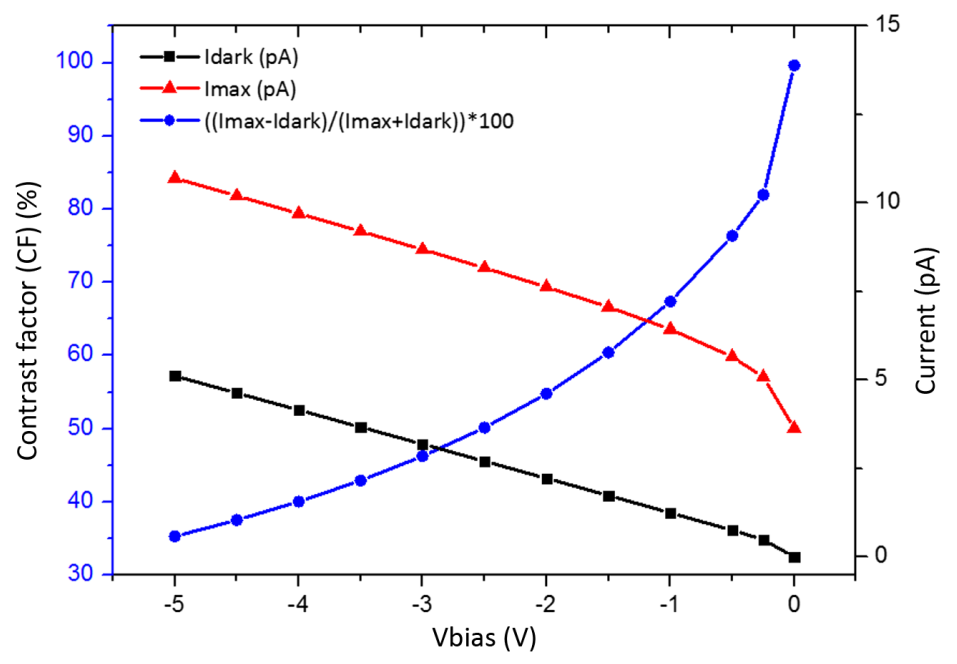

Fig. $7 I_{\text {max }}, I_{\text {dark }}$, and CF are all presented a function of the applied $V_{\text {bias }}$, based on Fig. 6 .

extract the detector's selectivity to the photocurrent, we define the detector's contrast factor $(\mathrm{CF})$ as

$$
\mathrm{CF}(\%)=100 \times \frac{I_{\max }-I_{\mathrm{dark}}}{I_{\max }+I_{\mathrm{dark}}}
$$

In Fig. 7, $I_{\mathrm{dark}}, I_{\max }$, and the CF are plotted as function of the bias voltage. The less is the bias voltage the more is the CF. An explanation will be developed later in the discussion.

\subsection{Photocurrent Scan as a Function of Radiant Intensity}

In order to evaluate the detector responsivity of the current, it was important to evaluate the influence of the laser beam parameters namely the radiant intensity and wavelength. In Fig. 8, the photocurrent scan was simulated as a function of several orders of magnitude of the radiant intensity $\left(0.15\right.$ to $\left.15 \mathrm{~W} / \mathrm{m}^{2}\right)$. Surprisingly, the "dark" current (located at $\pm 2 \mu \mathrm{m}$ ) is also sensitive to the radiant intensity. The beam seems to be larger at higher power. 


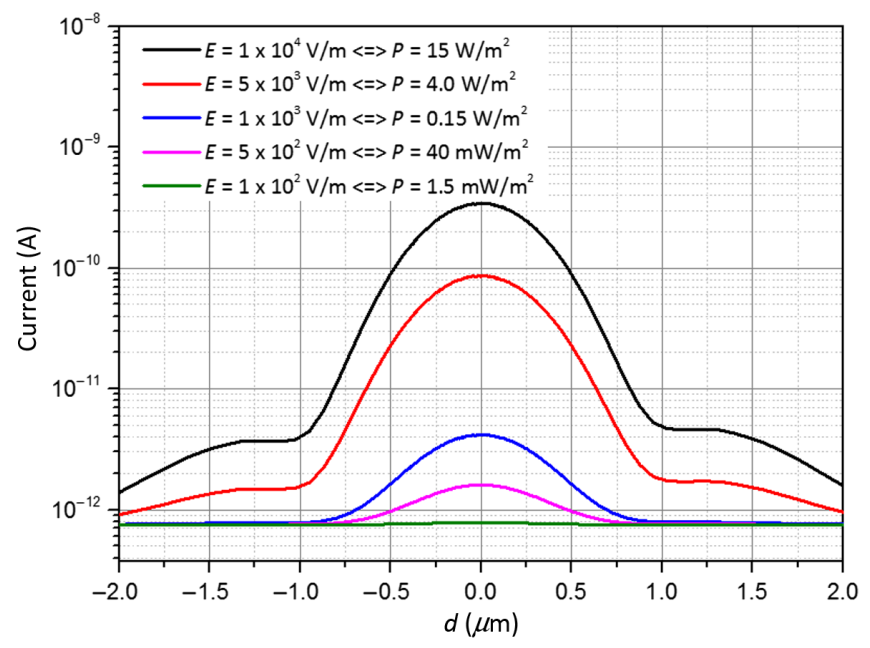

Fig. 8 Semilog plot of the photocurrent as a function of the photodetector's position $(\mu \mathrm{m})$, relative to the beam center, for several radiant intensity values. The simulation was performed under the following conditions: silicon doping is $10^{17} \mathrm{~cm}^{-3}$, work function is $4.72 \mathrm{eV}(\mathrm{Al}), \lambda$ is $550 \mathrm{~nm}$, and the voltage bias is $-0.5 \mathrm{~V}$.

It is conceivable that a larger beam illuminates the trapezoid from the sides below the metal contact, and this contributes to a higher "dark" current.

As expected, the increase of the radiant intensity implies a linear increasing of the current. Also, the photocurrent response should be consistent with realistic laser intensities as encountered in AFM systems. Of course, additional factors should be taken in account, including different types of decay, absorption, and quantum efficiency.

\subsection{Photocurrent Scan as a Function of Wavelength}

Now for a given radiant intensity of $0.15 \mathrm{~W} / \mathrm{m}^{2}$, the photocurrent response was studied for wavelengths ranging between 400 and $1100 \mathrm{~nm}$, by steps of $100 \mathrm{~nm}$. Several interesting characteristics are notable in Fig. 9. From 400 to $600 \mathrm{~nm}$, the current values are seen to steadily increase with the wavelength. However, jumps of the current are observed at 800 and $1000 \mathrm{~nm}$ revealing an oscillating phenomenon. The study was limited to $1100 \mathrm{~nm}$,

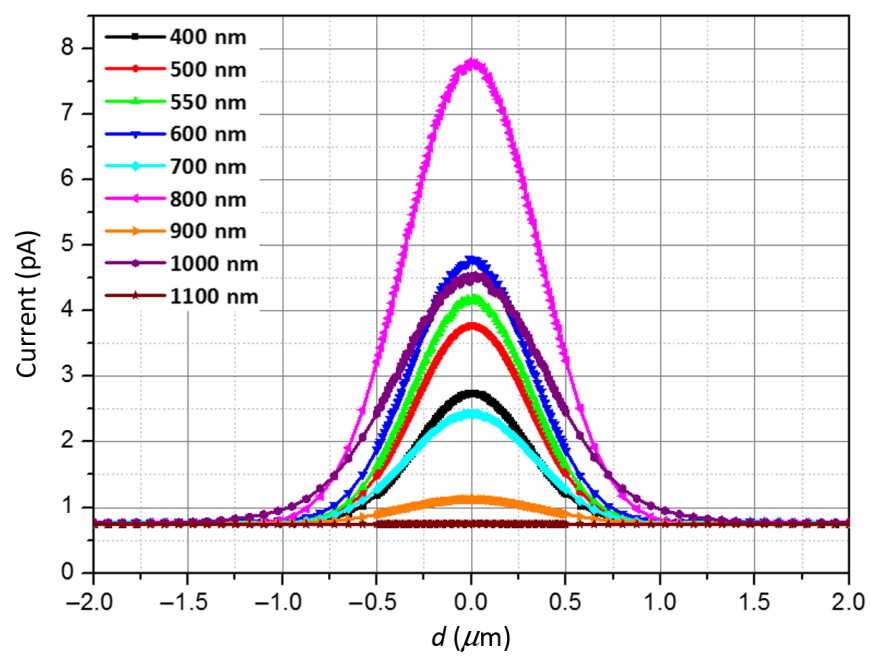

Fig. 9 Current as a function of the photodetector's position $(\mu \mathrm{m})$, relative to the beam center, for several wavelength values. The simulation was performed under the following conditions: doping is $10^{17} \mathrm{~cm}^{-3}$, work function is $4.72(\mathrm{Al})$, the voltage bias is $-0.5 \mathrm{~V}$, and the radiant intensity is $0.15 \mathrm{~W} / \mathrm{m}^{2}$. 
corresponding to nominal range, in which the absorption of silicon is significant. A further analysis will be presented below in order to elucidate this point.

\section{Analysis and Discussion}

\subsection{Semiquantitative Model of the Photocurrent}

Assuming the photocurrent is due primarily to the drift of the photogenerated carriers in the depleted layer and assuming a one-dimensional model for simplicity sake, the steady state photocurrent density $J_{\mathrm{ph}}$ (per unit area of the electrode area) is expected to increase with the depletion layer width $w$ like lin $^{20}$

$$
J_{\mathrm{ph}}=q \int_{0}^{w} G_{\mathrm{ph}}(x) \mathrm{d} x=q G_{\mathrm{ph}}(0) \frac{1}{\alpha}\left(1-e^{-\alpha w}\right)
$$

where $q$ is the electron charge, $G_{\mathrm{ph}}(x)$ is the volume photogeneration rate proportional to the radiant intensity, and $\alpha$ is the absorption coefficient of silicon, which is the function of the wavelength. Assuming the Schottky is a one-sided abrupt junction, $w$ decreases as the square root of doping level $N_{D}$ and increases as the square root of reverse bias $V_{\text {rev }}$ according the following equation:

$$
w_{(\mathrm{nm})}=\sqrt{\frac{2 \epsilon_{s} \epsilon_{0}}{q N_{D}}\left(V_{\mathrm{bi}}+\left|V_{\mathrm{rev}}\right|\right)} \sim 114 \sqrt{V_{\mathrm{bi}}+\left|V_{\mathrm{rev}}\right|}
$$

With $\epsilon_{s}$ is the silicon relative permittivity $(=11.7)$ and $N_{D}$ is the silicon doping $\left(10^{17} \mathrm{~cm}^{-3}\right)$. $V_{\mathrm{bi}}$ is the built-in voltage of the Schottky diode defined as the difference between the Fermi levels of the metal and the silicon. However, the $w$ value is limited here by the detector aperture of $150 \mathrm{~nm}$. Above this limit, the depletion areas from both sides of the metal contact are reaching each other and no further expansion is possible. This may explain why the CF of the photocurrent is decreasing while increasing $V_{\text {rev }}$ above $1 \mathrm{~V}$ as shown in Fig. 7.

If the energy level reference is taken at the vacuum level, $V_{\mathrm{bi}}$ can be expressed as the difference between the silicon Fermi level $E_{F, n}$ and the metal Fermi level $E_{F, \text { metal }}=-\Phi_{m}$ according to

$$
V_{\mathrm{bi}}=\frac{1}{q}\left(E_{F, \mathrm{n}}-E_{F, \text { metal }}\right)=\frac{1}{q}\left(E_{F, n}+\Phi_{m}\right) .
$$

The silicon Fermi level is given by

$$
E_{F, n}=-\chi_{\mathrm{Si}}+k T \ln \frac{N_{D}}{N_{C}}
$$

Thus we get

$$
V_{\mathrm{bi}}=\frac{1}{q}\left(\Phi_{m}-\chi_{\mathrm{Si}}+k T \ln \frac{N_{D}}{N_{C}}\right)
$$

where $\chi_{\mathrm{Si}}=4.05 \mathrm{eV}$ is the electron affinity of silicon, or equivalently the depth of the edge of the conduction band edge $E_{C}$. Using the work function value of aluminum $\left(\Phi_{m}=4.72 \mathrm{eV}\right)$ and the density of states value for silicon at $300 \mathrm{~K}\left(N_{C}=2.8 \times 10^{19} \mathrm{~cm}^{-3}\right)$, we get a value for $V_{\mathrm{bi}}$ of $0.543 \mathrm{~V}$. Replacing $V_{\text {bi }}$ by its value in Eq. (3) and taking into account the limitation on $w$ of $83 \mathrm{~nm}$, one finds a limiting value for $V_{\text {rev }}$ of about $-0.5 \mathrm{~V}$. This is consistent with Fig. 7, in which the currents depend on (the absolute value of) $V_{\text {rev }}$ as a square root like function below $-1 \mathrm{~V}$ and as a linear function above $-1 \mathrm{~V}$. The linear trend of the current with the voltage bias is due to the drift of photogenerated carriers. 
Since the dark current is zero at zero voltage (the thermal generation current is negligible), it turns out that the CF is maximum (100\%) at zero voltage and decay to a constant value as shown in Fig. 7. This semiquantitative model is helpful to interpret the increase of the simulated current with the decreasing of the doping level as shown in Fig. 4. Moreover, according to Eqs. (3) and (5), the depletion width $w$ increases for metals with larger work function, with a square root behavior at low voltage bias. This indeed is borne out in Fig. 5, where it can be observed that the three elements show values of current, which increase with the value of their respective work function.

\subsection{Responsivity}

As suggested from Fig. 8, one of the most important results expected from our simulation is a pure linear relation between the maximum value of the photocurrent $(d=0 \mu \mathrm{m})$ and the irradiance, i.e., the radiant intensity per unit area of the detector surface. When developing electrooptical devices and in particular nanoscale-based sensors, such a linear relation is of considerable importance since it allows easy prediction of the current produced for any desired irradiance. In Fig. 10, the plot of this relation is clearly displayed.

In order to evaluate the responsivity of the photodetector, it was important to quantify the electrical field from the Gaussian laser beam, outside and inside the photodetector, through the trapezoid aperture. The responsivity is the ratio between the current and the irradiance. We need to estimate it from the slope of Fig. 10 and get around $0.66 \mathrm{pA} / \mathrm{W} / \mathrm{m}^{2}$.

Figure 11 shows the vertical electrical field on top of the upper surface of the trapezoid. Figure 12 shows its simulated values of the electric field all along the vertical line centered at $d=0 \mu \mathrm{m}$ (no lateral shift). The horizontal red line represents the height ( $h=-0.5 \mu \mathrm{m}$ ), in which the trapezoid's top aperture stands and defined as an insulating boundary condition. Between the field's upper value of $1480 \mathrm{~V} / \mathrm{m}$ (just above the aperture) and the field's lower value of $600 \mathrm{~V} / \mathrm{m}$ (just under the aperture), there is a ratio of $40 \%$, which is a significant value. A clear distinction appears between the propagating field and the evanescent field at the detector aperture (150-nm diameter).

\subsection{Spectral Response}

The last interesting check was to evaluate the behavior of the photocurrent as a function of the wavelength, this time for several aperture diameters $(A=150,300,500 \mathrm{~nm})$. As observed in Fig. 13, the smaller the aperture is, the lower the current is. Also, there are two distinct areas: the

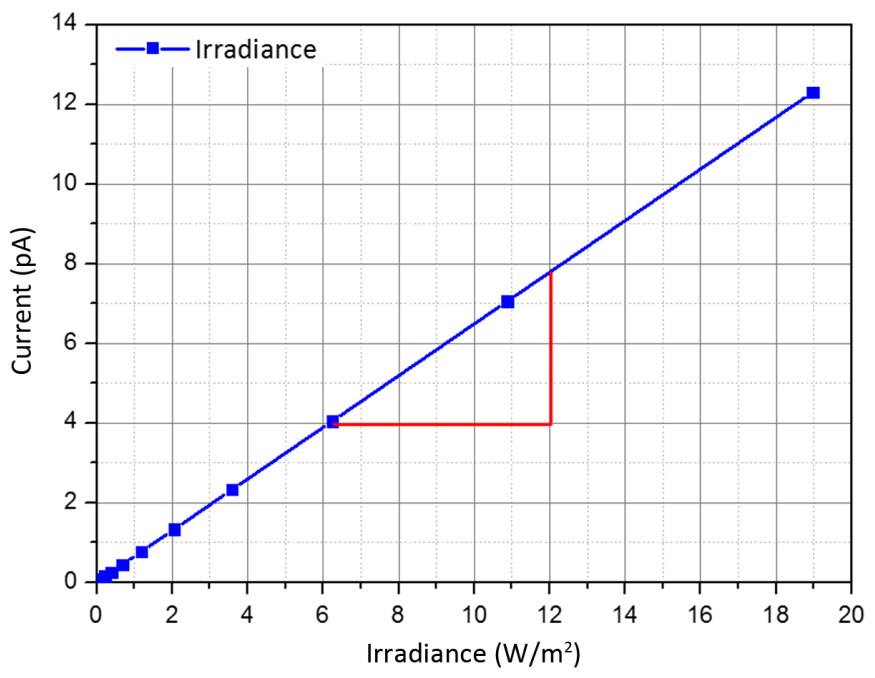

Fig. 10 Photocurrent as a function of the irradiance. The simulation was performed under the following conditions: Silicon doping is $10^{17} \mathrm{~cm}^{-3}$, work function is $4.72 \mathrm{eV}(\mathrm{Al}), \lambda$ is $550 \mathrm{~nm}$, and the voltage bias is $-0.5 \mathrm{~V}$. 


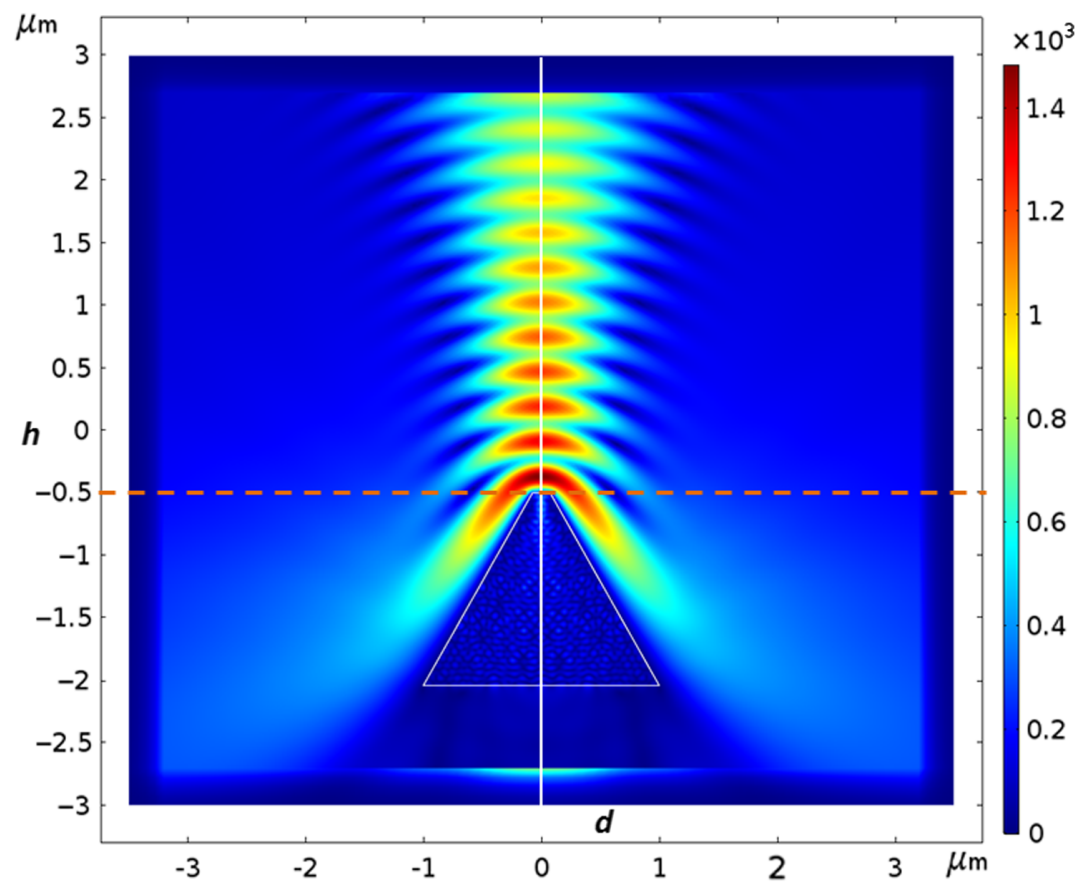

Fig. 11 Electrical field outside and inside the photodetector. The border at the aperture's height is represented by the horizontal red line. The vertical line is the path, in which the field values have been measured in Fig. 12.

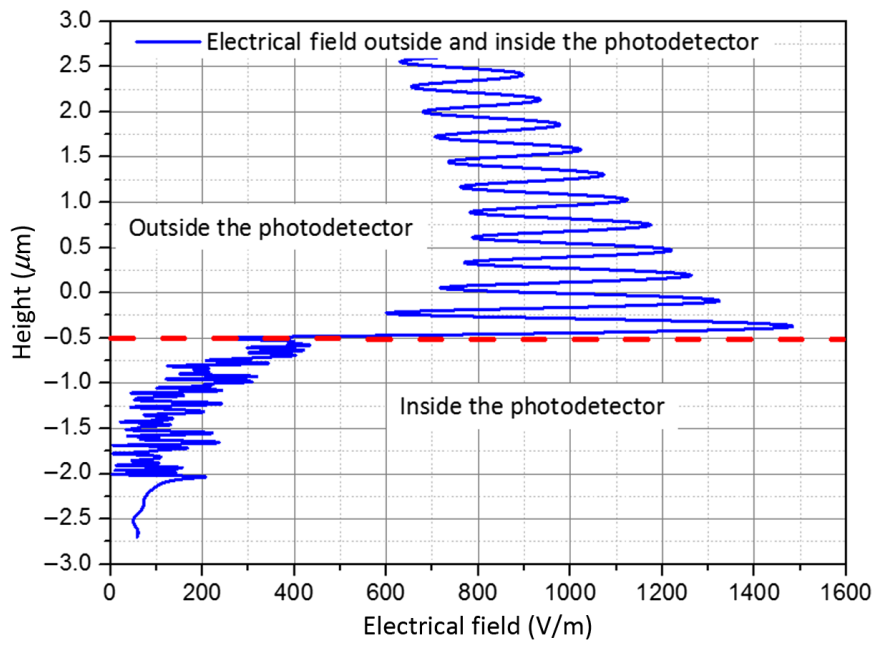

Fig. 12 Laser beam electrical field outside and inside the photodetector. The border at the aperture's height is represented by the horizontal red line. Aperture diameter is $150 \mathrm{~nm}$ and the radiant intensity is $0.15 \mathrm{~W} / \mathrm{m}^{2}$.

left one, till $\lambda=600 \mathrm{~nm}$, where the current keeps the same shape of oscillations, and decreasing with smaller aperture diameters. However, the right area shows some interference pattern but the current is less sensitive to the aperture diameter. The attenuation of the current at very short values of $\lambda(<450 \mathrm{~nm})$ is due to strong absorption of light at silicon surface. We can see that, in addition to this diffraction phenomenon, there is also an interference additional phenomenon, shown in the right side of this figure; these oscillations may be due to the fact that part of the electrical field is reflected by the detector, and interfering with the original laser beam as simulated in Fig. 11. 
Karelits et al.: Laser beam scanning using near-field scanning optical microscopy...

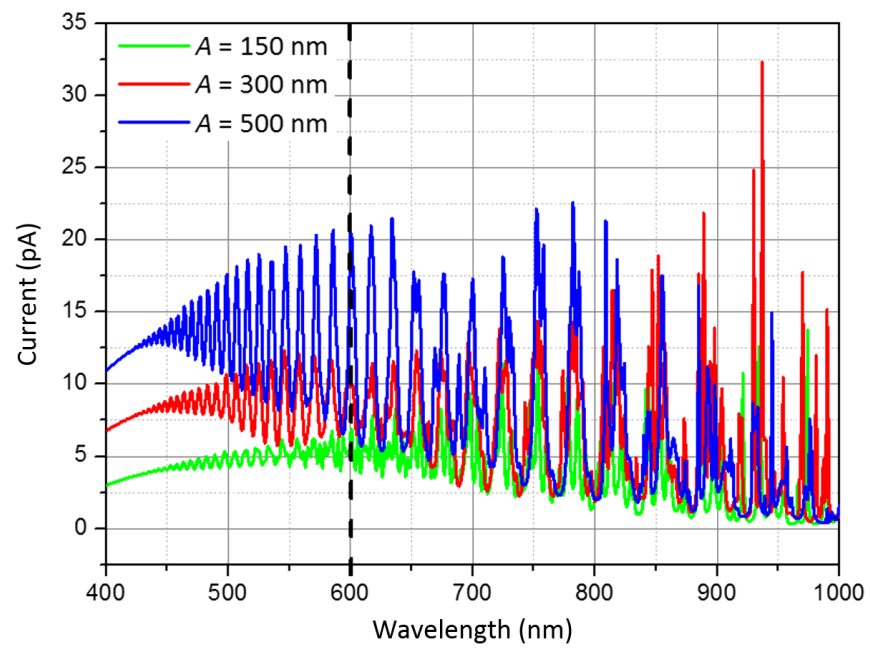

Fig. 13 Current as a function of the wavelength for several top aperture diameters. The simulation was performed under the following conditions: silicon doping is $10^{17} \mathrm{~cm}^{-3}$, work function is $4.72 \mathrm{eV}(\mathrm{Al})$, the voltage bias is $-0.5 \mathrm{~V}$, and the radiant intensity is $0.15 \mathrm{~W} / \mathrm{m}^{2}$.

Table 1 Recommended parameters of the photodiode and of the setup.

\begin{tabular}{lcc}
\hline \hline Sign & Definition & Recommended value \\
\hline Device's intrinsic specifications & & \\
$D$ & Doping concentration & $10^{16} \mathrm{~cm}^{-3}$ \\
$\Phi_{m}$ & Metal work function & $4.72 \mathrm{eV}(\mathrm{Al})$ \\
$A$ & Top aperture diameter & $150 \mathrm{~nm}$ \\
Setup external parameters & & \\
$V$ & Bias applied voltage & $-0.5 \mathrm{~V}$ \\
$P$ & Radiant intensity & $0.15 \mathrm{~W} / \mathrm{m}^{2}$ \\
$\lambda$ & Wavelength & $>600 \mathrm{~nm}$ \\
\hline \hline
\end{tabular}

\subsection{Optimization and Recommended Setup Values}

Series of above simulations and analyses enable to forecast and optimize the behavior and the accuracy of the NSOM photodetector for two different series of parameters: the device's specifications (doping, work function, etc.) and the setup parameters (external electrical field, wavelength, etc.). This is why it was important to summarize all the recommended values in Table 1. Summarizing above analyses, it appears that the highest measured current was obtained for a doping of $10^{16} \mathrm{~cm}^{-3}$ (Fig. 4), for a work function of gold (Fig. 5) and a wavelength above $600 \mathrm{~nm}$ (Fig. 13). Regarding the work function's preference, if the gold enables a higher current, the aluminum is more suitable to work with silicon and is usually preferable from an evaporation process point of view since less contaminating. This is why we kept the choice of aluminum in Table 1. A low reverse bias $(-0.5 \mathrm{~V})$ is recommended to get a high $\mathrm{CF}$ of the photocurrent (Fig. 7).

\section{Conclusion}

In this paper, we have presented the electro-optical scanning of a vertical laser beam, using a shifted photodetector with subwavelength aperture, in front of an illumination beam. This study consisted in varying critical parameters-including the applied bias voltage $(V)$, the doping 
concentration $(D)$, the power of the light illumination intensity $(P)$, the wavelength $(\lambda)$, the top diameter aperture $(A)$, and the metal contact work function $\left(\Phi_{\mathrm{m}}\right)$-in order to find the optimal scanning conditions for integration of this variety of photodetector on a cantilever. If several studies presented some ideas of photodetectors for NSOM purpose, this study enables an easy forecast of the expected behavior of the device, as a function of internal and external parameters.

\section{References}

1. L. Salomon et al., "Near-field distribution of optical transmission of periodic subwavelength holes in a metal film," Phys. Rev. Lett. 86, 1110-1113 (2001).

2. T. Ishi et al., "Si nano-photodiode with a surface plasmon antenna," Jpn. J. Appl. Phys. 44(Part 2), 12-15 (2005).

3. F. Pavanello, "Uni-travelling carrier photodiodes and metal mesh filters based on sub-wavelength apertures for THz applications," PhD Thesis, Université des Sciences et Technologies de Lille (2013).

4. R. C. Davis, C. C. Williams, and P. Neuzila, "Micromachined submicrometer photodiode for scanning probe microscopy," Appl. Phys. Lett. 66(18), 2309-2311 (1995).

5. G. Strang and G. Fix, An Analysis of the Finite Element Method, 2nd ed., WellesleyCambridge Press, Wellesley, Massachusetts (2008).

6. A. Karsenty and Y. Mandelbaum, "Computer algebra challenges in nanotechnology: accurate modeling of nanoscale electro-optic devices using finite elements method," accepted to Math. Comput. Sci..

7. R. A. Chelly et al., "Pyramid-shaped silicon photodetector with subwavelength aperture," IEEE Trans. Electron Devices 49(6), 986-990 (2002).

8. M. Karelits et al., "Nanoscale silicon truncated conical photodetector at subwavelength aperture for NSOM applications," in Proc. IEEE Int. Conf. Optical MEMS and Nanophotonics (OMN), Santa Fe, New Mexico, USA, 13-17 August (2017).

9. M. Karelits et al., "Electro-optical study of nanoscale Al-Si truncated conical photodetector with subwavelength aperture," J. Nanophotonics 11(4), 1 (2017).

10. C. H. Liou et al., "Design and fabrication of a near-field scanning optical microscope probe by CMOS-MEMS," in IEEE Int. Conf. Optical MEMS and Nanophotonics, 6-9 August (2012).

11. L. Zhou et al., "Direct near-field optical imaging of UV bowtie nanoantennas," Opt. Express 17, 20301-20306 (2009).

12. N. Perney et al., "Tuning localized plasmons in nanostructured substrates for surfaceenhanced Raman scattering," Opt. Express 14, 847-857 (2006).

13. A. Alabastri et al., "Super-lattice effects in ordered core-shell nanorod arrays detected by Raman spectroscopy," in Proc. COMSOL Conf., Stuttgart (2012).

14. I. Knorr et al., "Prediction and optimization of surface-enhanced Raman scattering geometries using COMSOL multiphysics," in Proc. of the COMSOL Conf., Hannover (2008).

15. N. C. Linn et al., "Surface-enhanced Raman scattering on periodic metal nanotips with tunable sharpness," Nanotechnology 20(22), 225303 (2009).

16. A. Downes, D. Salter, and A. Elfick, "Finite element simulations of tip-enhanced Raman and Fluorescence Spectroscopy," J. Phys. Chem. B 110(13), 6692-6698 (2006).

17. M. A. Green and M. J. Keevers, "Optical properties of intrinsic silicon at $300 \mathrm{~K}$," Prog. Photovoltaics Res. Appl. 3, 189-192 (1995).

18. L. Novotny and B. Hecht, Principles of Nano-Optics, Cambridge University Press, Cambridge, United Kingdom (2006).

19. C. Kittel, Introduction to Solid-State Physics, 8th ed., Wiley, Hoboken, New Jersey (2004).

20. S. Sze and M. Lee, Semiconductor Devices: Physics and Technology, 3rd ed., John Wiley \& Sons Singapore Pte. Limited, Singapore (2012).

Matityahu Karelits started studies toward his BSc degree in applied physics/electro-optics engineering three years ago, at the Lev Academic Center (formerly JCT-Jerusalem College of Technology). He is also active member of the Advanced Laboratory of Electro-Optics (ALEO), led by Dr. Avi Karsenty. 
Yaakov Mandelbaum studied mathematics and physics at the undergraduate and graduate levels at the University of Pennsylvania, MIT, and the Hebrew University of Jerusalem. After working in industry as a physicist and electro-optics team leader, he joined the Lev Academic Center in 2013 as a lecturer and researcher in the Department of Applied Physics and Electro-Optics. In parallel, he is currently pursuing his $\mathrm{PhD}$ at Bar-Ilan University.

Avraham Chelly received his $\mathrm{PhD}$ in solid-state physics from the Université de Haute Alsace, Mulhouse, France, in 1997. During his postdoc at the Hebrew University of Jerusalem, Israel, he was involved in the development and fabrication of silicon nanodetector for near-field optics. In 2004, he moved to Bar-Ilan University, where he established the Advanced Semiconductor Devices Laboratory. He is giving lectures on semiconductor processing and devices.

Avi Karsenty received his $\mathrm{PhD}$ in applied physics/material science (microelectronics/electrooptics) from the Hebrew University of Jerusalem in 2003. His research focuses on nanoscale electro-optics coupled-devices. After 22 years in High-Tech industries, part of which as an engineer and a manager for 16 years with Intel, he is currently the head of the Physics/Electro-Optics Engineering Department. He is an IEEE senior member and an OSA senior member, and received 40 awards in engineering/physics. 